Tohoku J. exp. Med., 1965, 84, 316-328

\title{
Cerebral Circulation during Arousal Reaction of EEG
}

\author{
By \\ Hisao Suzuki and Yasuo Tukahara \\ From the Institute of Brain Diseases, Tohoku University \\ School of Medicine, Sendai
}

(Received for publication, August 14, 1964)

\begin{abstract}
The vascular behavior of various areas in the cortex and thalamus during arousal reaction of EEG was studied in locally anesthetized and with Flaxedil immobilized cats. For inducing the arousal reaction, iterative rectangular pulses were applied to the mesencephalic reticular formation, and for measuring local blood flow in the brain, the heated thermistor method described previously was utilized. In the cat with the intact central nervous system, the arousal reaction accompanied always a temporal rise in the systemic blood pressure. In parallel with this pressor response, increases in blood flow occurred, both in cortical and thalamic areas. In the encéphale isolé cat, EEG arousal appeared without any observable change of blood pressure. Despite the lack of the pressor response in this preparation, EEG arousal induced a definite flow increase in the thalamic areas lasting for over $30 \mathrm{sec}$, whereas the local blood flow in the cerebral cortex remained unchanged during the reaction as far as it was measured by the heated thermistor method.

When i.v. injection of Nembutal was made, both EEG and thalamic flow response to MRF stimulation diminished concurrently. Stimulation to VA and $\mathrm{CM}$ also evoked similar flow responses.
\end{abstract}

The vascular behavior of the cerebral cortex during the arousal reaction of EEG was studied by Ingvar using a perfusion technique of the superior sagittal sinus, and it was reported that a definite increase in cortical blood flow occurred with the arousal reaction ${ }^{4}$. However, his observation has not been confirmed with techniques which were devised for measuring more localized vascular changes in the brain tissue ${ }^{2,5,6)}$. The purpose of the present investigation is to measure local blood flow in the brain during the arousal with the heated thermistor method described previously').

\section{METHODS}

All experiments were carried out on locally anesthetized and with Flaxedil immobilized cats. Under ether anesthesia, the trachea was cannulated, a polyethylene tubing was inserted into the saphenous vein and the head was

鉿木寿夫，塚原保夫 
fixed to a stereotaxic apparatus. The pial surface of the cerebral cortex was exposed in the routine procedure. Thereafter, stimulating and recording electrodes and a sensing element for measuring local blood flow were set in the brain. Throughout the experiment, artifical respiration was maintained.

For making the encéphale isolé preparation, the spinal cord was exposed at $\mathrm{C}_{\mathbf{1}}$ by removing the atlanto-occipital membrane and was transected with a blunt spatula. After the transection at least $30 \mathrm{~min}$ were allowed to pass before the commencement of experiment.

For inducing the arousal reaction of EEG, a train of monophasic rectangular pulses was applied to the mesencephalic reticular formation (MRF: F 1, L 3, H -2) through bipolar stimulating electrodes when otherwise not mentioned. Usually its frequency was $200 / \mathrm{sec}$ and duration of each pulse was $1 \mathrm{msec}$. The stimulating electrodes used consisted of two stainless steel wires of $100 \mu$ insulated except at the tips and cemented together so that tip separation was $1 \mathrm{~mm}$.

In order to measure local blood flow in the cortical and subcortical structures, we adopted the procedure described previously in detail ${ }^{9}$. So, only a brief summary of the method will be given here. A needle-type element having a thermistor bead on its tip is inserted into a part of the brain. The bead which is heated by a steady electric current, is cooled by blood flow around it, the rate of cooling being proportional to the flow rate. Since the electric resistance of the thermistor bead varies with the cooling, such a change in resistance can be used as an index of the flow rate.

Arterial blood pressure was measured continously from the femoral artery by means of a strain gauge transducer. The electrocorticogram (ECG) was taken through silver ball electrodes placed on the cortical surface. The electrocorticogram, local bood flow and arterial blood pressure were all recorded simultaneously with an ink-writing pen recorder.

At the end of the experiment, the brain was removed and hardened in 10 per cent formalin for identification of the place where the blood flow was recorded with the thermistor element. The stereotaxic atlases of Jasper and Ajmone-Marsan. were used as guides.

\section{RESULTS}

\section{Arousal reaction of EEG and pressor response to electrical stimulation of $M R F$}

The effect of stimulating the MRF on the ECG and on the systemic arterial pressure of the cat with the intact central nervous system is illustrated in Fig. 1A. As can be seen in the lower tracing of this figure, the resting ECG obtained from the pericruciate gyrus represented irregular low voltage activity mixed with large slow waves. The systemic arterial pressure, measured from the femoral artery, ranged between 120 and $160 \mathrm{~mm} \mathrm{Hg}$ maintaining a relatively steady 


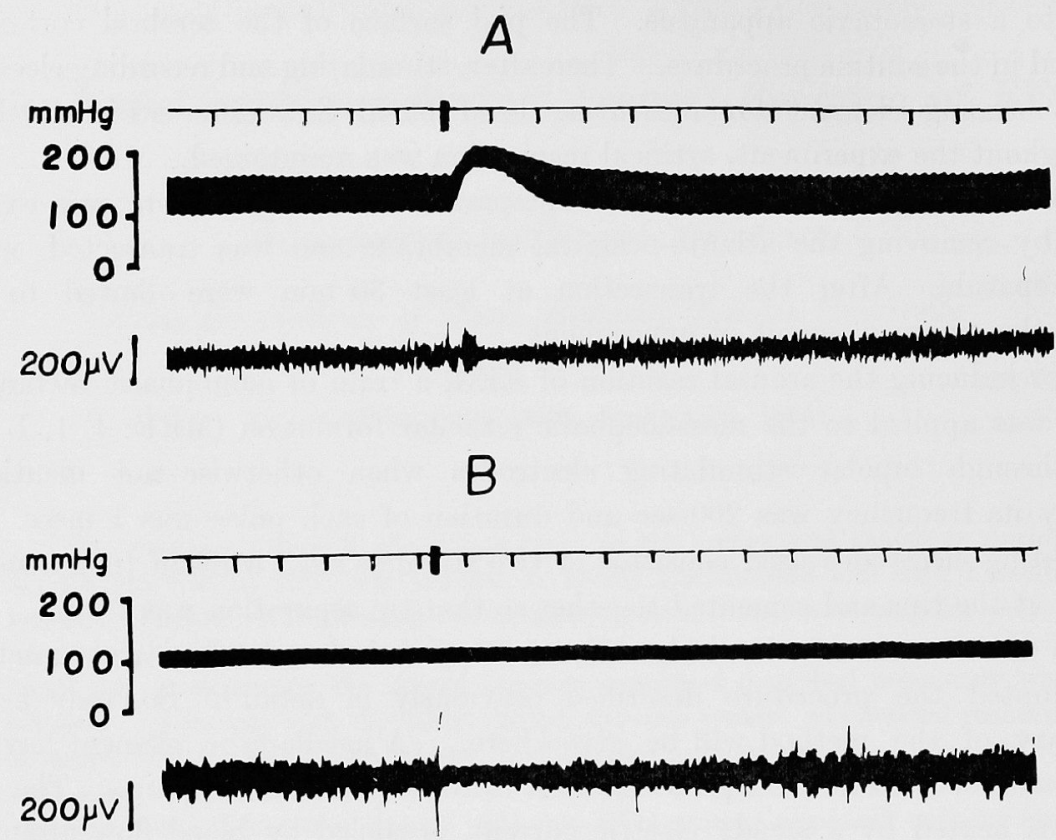

Fig. 1. Effect of MRF stimulation on systemic blood pressure and ECG. A: Intact animal. Repetitive stimulation (200/sec, $1 \mathrm{msec}, 5 \mathrm{~V}$ for $2 \mathrm{sec}$ ) to MRF evoked marked EEG arousal and concomitant pressor response. From top to bottom; time marker of $10 \mathrm{sec}$ and stimulus mark, systemic blood pressure and ECG of postcruciate gyrus. B: Cat after section at $\mathrm{C}_{1}$ (encéphale isolé cat). Stimulus parameters are same as A. Note marked EEG arousal without any pressor reaction.

value except for superimposed waves synchronous with the heart beat and respiratory movement (upper tracing). Application of high frequency stimulation ( $5 \mathrm{~V}$ for $2 \mathrm{sec}$ ) to the MRF resulted in EEG arousal, that is, the appearance of irregular low voltage activity which persisted for at least several seconds. Such EEG arousal was always accompanied by a temporal rise in the systemic blood pressure which began with a latency of a few sec, reached maximum in 5 to 10 sec and waned gradually to the original value in 20 to 50 sec.

Disection at $\mathrm{C}_{1}$ level of the spinal cord caused a considerable decrease of the systemic blood pressure as shown in the upper tracing of Fig. 1B; under this condition the blood pressure ranged from 50 to $120 \mathrm{~mm} \mathrm{Hg}$. In the resting ECG, large slow waves became predominant (lower tracing). Reticular stimulation, parameters of which were similar to those for the intact animal still gave rise to definite desynchronization of the ECG lasting for several seconds. However, it failed to produce any significant modification in the blood pressure (see lower tracing). This lack of pressor response during the arousal in the encéphale isolé preparation is well in line with the results obtained by Ingvar ${ }^{4}$. 
Local blood flow in brain during EEG arousal induced by reticular stimulation

MRF stimulation increased both cortical and thalamic blood flow in the intact animal and these flow increases occurred in parallel with elevation of the blood pressure which was always observed in this preparation on MRF stimulation (cf. Fig. 1A). An example of flow responses of the cortex and thalamus of an intact animal is illustrated in Fig. 2. In this figure the depth of the sensing element in the brain is indicated by ordinate of the F 9-L 6 line of the stereotaxic atlases. As can be seen in the uppermost tracing representing the cortical blood flow, some spontaneous fluctuation was observed in the resting state, and on MRF stimulation a definite increase of flow occurred over about 2 minutes. Responses of the same type were obtained from the pericruciate, suprasylvian and lateral gyri.

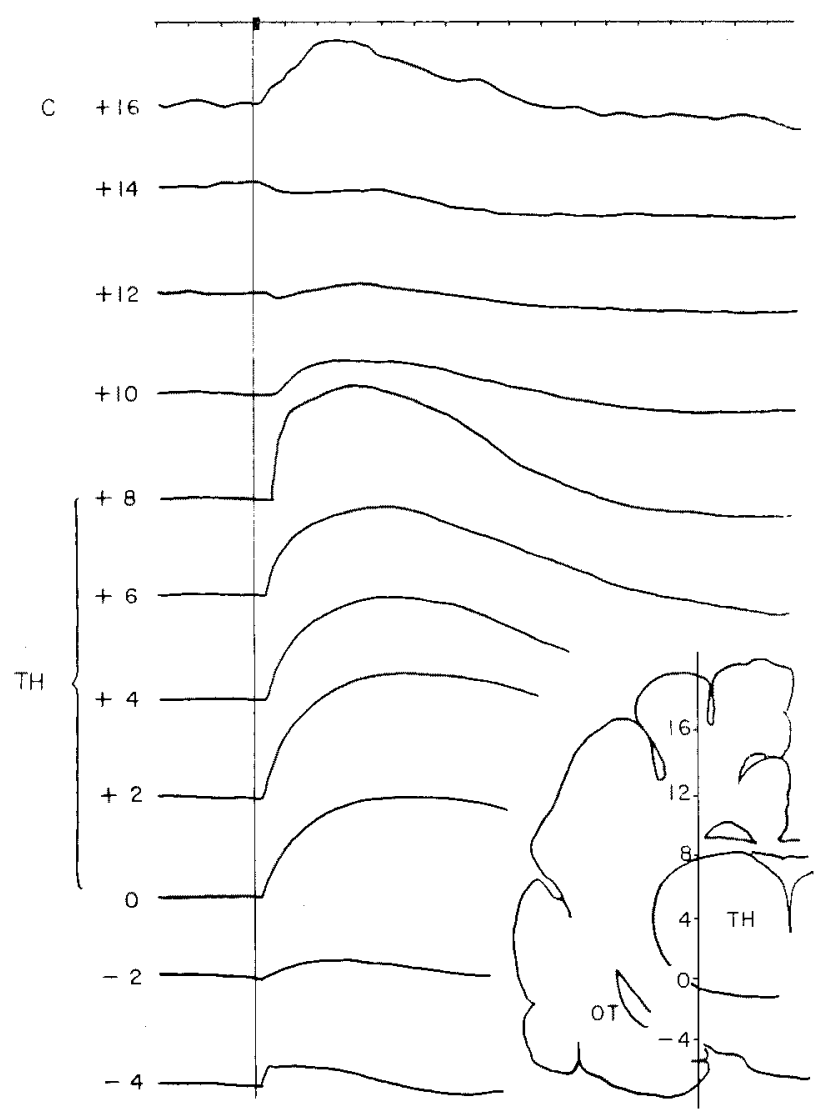

Fig. 2. Blood flow responses to MRF stimulation at various depths from cortex of intact cat. Time marker 10 sec. Vertical line indicates moment at which stimulus was given. $\mathrm{C}$ : cortex. TH: thalamus. Upward deflection indicates increase of flow rate. Number beside each trace shows stereotaxic depth illustrated in inset. 
The flow in the thalamic regions also increased on MRF stimulation as illustrated in Fig. 2. Between the flow response of the cortex and that of the thalamus there were some differences: The magnitude of response was generally smaller in the cortex than in the thalamus, and moreover, there seemed to be some phase difference between the cortex and thalamus in such a way that the peak of the thalamic response tended to lag behind that of the cortical response. These findings seem to suggest that cortical vascular beds may have a better intrinsic regulatory mechanism than the subcortical structures.

In earlier series of the present experiments, it had been tried to insert the thermistor element into each nucleus of the thalamus for measurement of its local flow response, but such attempts were abandoned, because it was found that the thermistor element used was too large in size to assess such detailed local differences.

The transection of the spinal cord at $\mathrm{C}_{1}$ greatly modified the flow responses of the brain to MRF stimulation in addition to the described effect that the

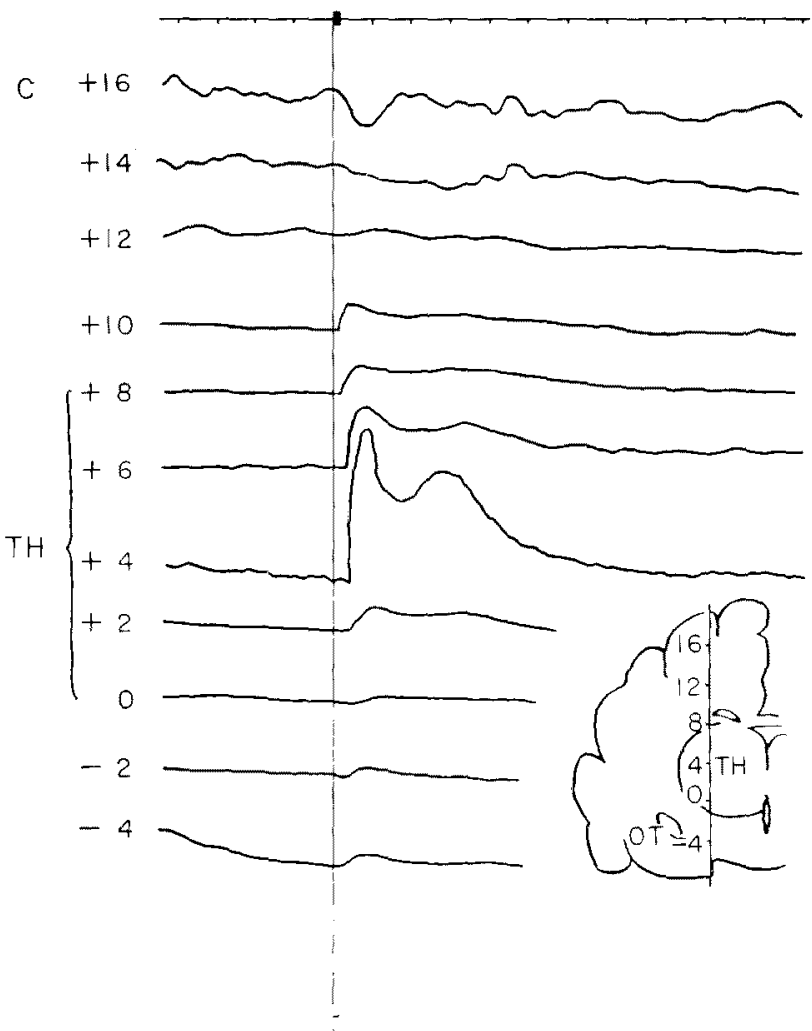

Fig. 3. Blood flow responses to MRF stimulation at various depths from cortex of encéphale isolé cat. Other explanations as in Fig. 2. 
procedure obliterated the pressor response. MRF stimulation of the encéphale isolé preparation increased significantly thalamic blood flow without any simultaneous increase in the arterial blood pressure. The effects of MRF stimulation on cortical and thalamic blood flow in the encéphale isolé preparation are illsutrated in Fig. 3. The cortical blood flow suffered no appreciable change, as can be seen in the uppermost tracing of this figure, but in the thalamus a remarkable response occurred to MRF stimulation. The response followed the appearance of activation pattern of EEG with a delay of a few seconds, and was characterized by its dicrotic pattern; the first wave was usually larger in amplitude and shorter in duration than the second one which persisted for over 30 seconds. It is to be noted that in the encéphale isolé preparation the cortical flow response fails to occur in association with the disappearance of the pressor response, whereas the thalamic response can be evoked as easily as in the intact animal.

The cortical flow showed some spontaneous fluctuation having an average period of 40-70 seconds as mentioned above, and even this fluctuation underwent little modification on MRF stimulation. On several occasions, however, intense reticular stimulation induced small but prolonged reduction in cortical blood flow.

\section{Stimulus strength and flow responses}

Fig. 4 represents a series of records of cortical blood flow in the encéphale

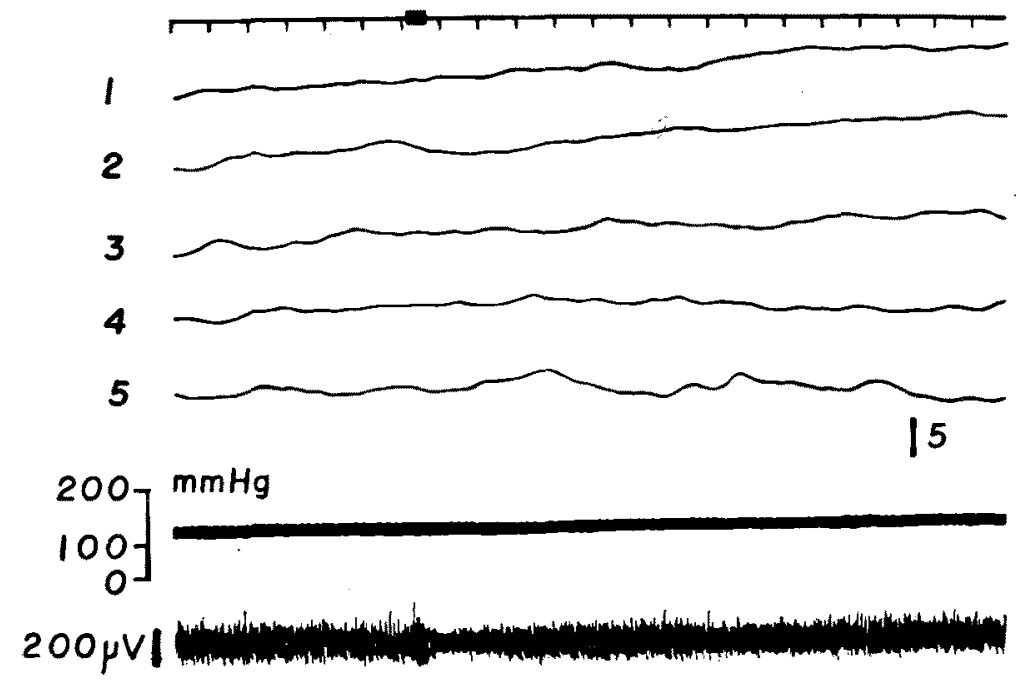

Fig. 4. Inactive behavior of vascular bed in cerebral cortex at various intensities of MRF stimulation (200/sec, $1 \mathrm{msec}$ for $5 \mathrm{sec}$ ). No response could be seen in suprasylvian gyrus. Numeral on left of each trace represents relative intensity of stimulus. Lowest two traces show systemic blood pressure and ECG of postcruciate gyrus which were recorded together with uppermost flow record (relative intensity 1). Calibration for flow responses is given by vertical line in relative units. 


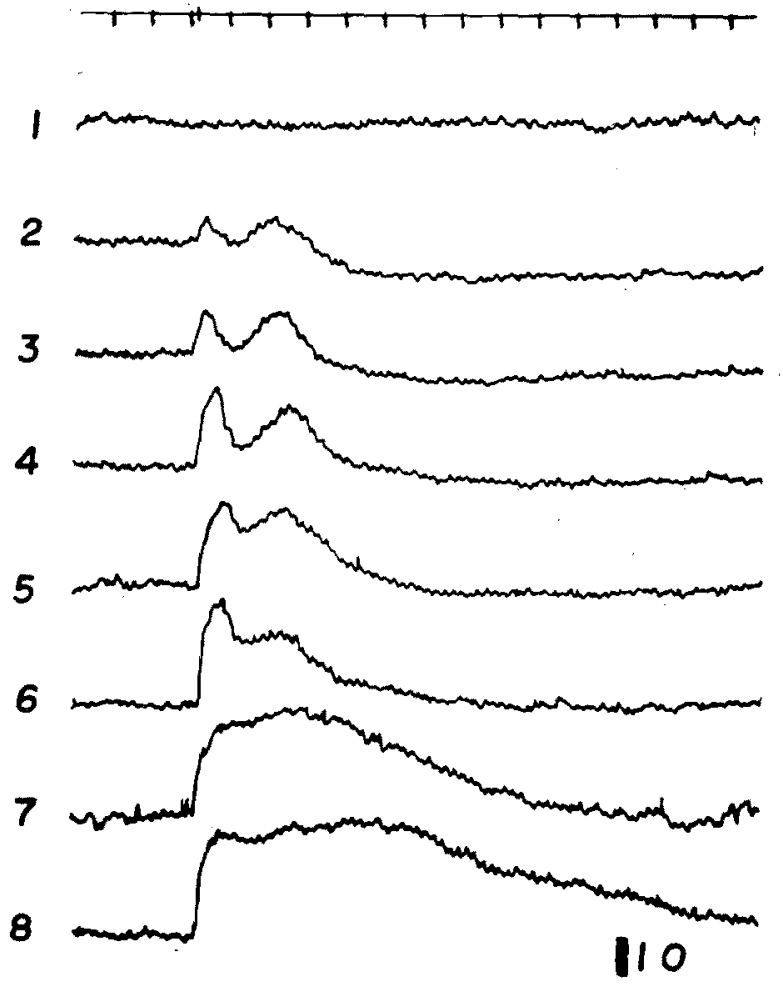

Fig. 5. Thalamic flow responses to stimuli of various intensities applied to MRF. Numeral on left of each trace represents relative intensity of stimulus. Calibration for flow responses is given by vertical line in relative units.

isole cat when stimuli of various intensities were applied to the MRF. In these experiments the amplitude of each pulse was changed, whereas its duration and frequency remained constant. As seen in this figure, there was no observable response in the cortical vascular bed irrespective of the very high intensity of stimulus used such as over five times the threshold for evoking EEG arousal. The minor variation in cortical blood flow observable in the records was spontaneous one occurring in very irregular fashion without any correlation to the stimulus.

In contrast to such an inactive behavior of the cortical circulation, the thalamic vascular response to reticular stimulation was highly dependent of stimulus intensities, and both amplitude and shape of the response changed according to the stimulus strength. With increase of the intensity, the magnitude of both first rapid and second slow components of the blood flow response increased (see Fig. 5). Moreover, the duration of the second component was prolonged and its magnitude relative to that of the first component was much increased so that both components became almost continuous (see 7 and 8 


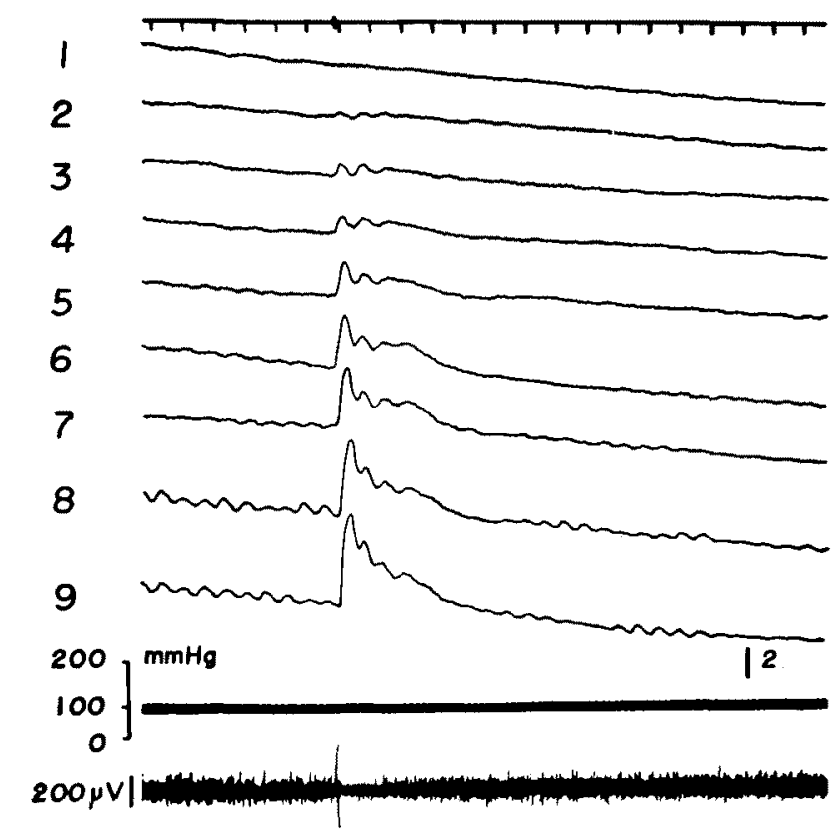

Fig. 6. Variant type of thalamic flow response to MRF stimulation. Stimulus parameters as in Fig. 5 .

in Fig. 5). In some cases, reticular stimulation induced such a variant type of flow response in the thalamus as shown in Fig. 6. In this type, the first peak was similar to the first component of the usual type, but jnstead of the second slow component of the usual type, multiple peaks appeared in the fashion of damped oscillations.

Effect of Nembutal on EEG arousal and vascular response in the thalamus

This experiment was concerned with the question as to whether or not Nembutal, an effective agent on the EEG activating system, affected also the vascular response accompanied with it. An example of the experiment is shown in Fig. 7. In this figure, each row represents a pair of records of thalamic blood flow and EEG taken simultaneously. Before intravenous injection of $20 \mathrm{mg}$ Nembutal, MRF stimulation induced a remarkable arousal reaction of EEG and thalamic flow increase (row 1). About 5 minutes after the injection, vascular and EEG responses were depressed slightly (see row 2). Then the second injection of the same amount was carried out to take simultaneous recording of both responses (row 3). After the third injection spontaneous electrical activity of the cortex became suppressed severely, and MRF stimulation was responded by appearance of the spindling of the EEG, but could not evoke any remarkable vascular response 

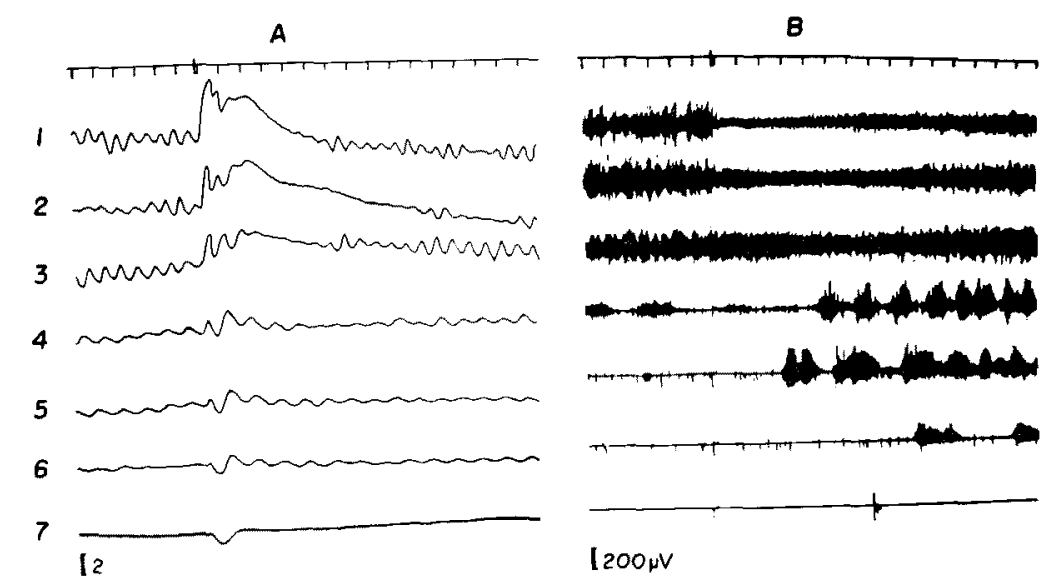

Fig. 7. Effect of Nembutal on EEG arousal and vascular response of thalamic area. A and B represent flow response and ECG of suprasylvian gyrus respectively. Flow response and ECG in each row were recorded simultaneously. 1: before injection of Nembutal. Nembutal of $20 \mathrm{mg}$ was injected before each of other records, 2,3... was taken. Time marker 10 sec.

(see row 4). It is clear that both arousal and vascular responses are suppressed by a small amount of Nembutal. With a larger dosis of the drug, i.e., $120 \mathrm{mg}$, the ECG disappeared completely, and the vascular response of the usual form was lost sight of, but curious to say, a simple downward deflection still appeared (see row 7).

In the flow records shown in Fig. 7, spontaneous fluctuation of the flow by the rate of about $8 / \mathrm{min}$ was observed. It seemed that such spontaneous fluctuation was more remarkable at later stages of a prolonged experiment, its amplitude augmenting steadily toward the end of the experiment.

Stimulation of various loci in brain and flow responses

In the above experiments only MRF stimulation was used for eliciting flow responses. Therefore, in the following experiments the stimulating electrodes were placed in various structures in the brain, the other conditions being the same as above. It was revealed by these experiments that thalamic flow responses of similar pattern and magnitude were obtained with stimulation of the nucleus ventralis anterior and the nucleus centrum medianum of the thalamus (VA and CM in Fig. 8B). In agreement with stimulation of the MRF, stimulation of these thalamic structures failed to evoke any definite vascular responses in the cerebral cortex (see VA and CM in Fig. 8A). Stimulation of the specific thalamic nuclei such as the ventrobasal complex and the nucleus ventralis lateralis seemed almost ineffective for inducing such flow responses. 
$A$

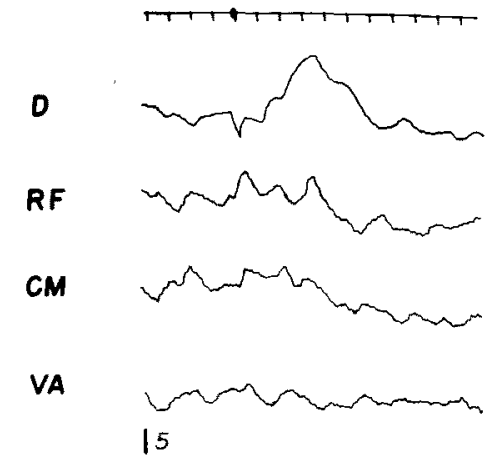

$B$

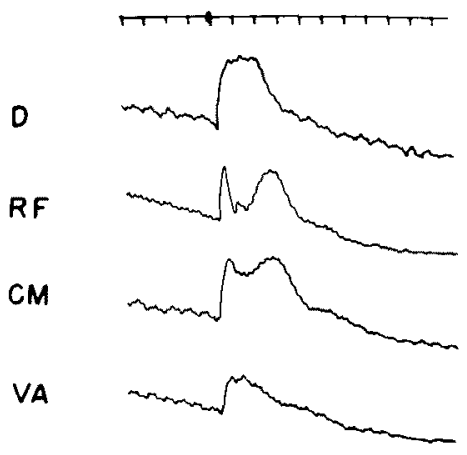

Fig. 8. Effects of stimulation applied to various loci. A and B represent flow records in cortex (suprasylvian gyrus) and thalamus respectively. D: direct stimulation of point where measurement of flow was made. RF: mesencephalic reticular formation. CM: nucleus centrum medianum. VA: nucleus ventralis anterior.

Direct stimultation of the structures from which the flow measurement was made produced a clear local increase in flow rate (D in Fig. $8 \mathrm{~A}$ and $\mathrm{B}$ ). In the cortex, however, the flow increase was converted into severe and prolonged reduction of the flow, as soon as the spreading depression was evoked by a strong stimulus.

\section{DISCUSSION}

The present experiments show that the local blood flow in the cerebral cortex of the encéphale isolé preparation undergoes no appreciable change with MRF stimulation. Although the method used was different from that in our experiments, Ingvar ${ }^{4}$ reported a quite different result that a definite increase in cerebral blood flow was induced by MRF stimulation. His experiments were done under rather abnormal condition such that a cannula was inserted into the superior sagittal sinus for measuring cerebral blood flow, and according to Carlyle and Grayson ${ }^{3}$ such a procedure might destroy the intrinsic regulatory power to keep the cortical blood flow constant, resulting in an increase of blood flow in response to MRF stimulation. If the postulate that the intrinsic regulatory mechanism is well developed in the cerebral cortex is correct, the observed absence of flow responses will be well understood. Kanzow et al. ${ }^{5,6)}$ who applied a disc-type thermoelectric element for measuring cerebral blood flow, indicated that the cortical blood flow did not relate primarily to EEG changes, and this relation applies also to our finding that no parallelism was found between EEG arousal and flow response at least in the encéphale isolé preparation. Birzis and Tachibana ${ }^{2)}$ also demonstrated the surprisingly inactive behavior of the cortex by using impedance changes measured with a high frequency bridge as an indicator for the rate-of blood flow. 
An alternative possibility to explain the Ingvar's result mentioned above would be that the blood flow in the superior sagittal sinus might be contaminated by the subcortical blood flow which was shown to increase definitely in response to MRF stimulation, although Ingvar $\left.{ }^{4}\right)$ insisted that the superior sagittal sinus collected exclusively cortical blood flow.

Birzis and Tachibana ${ }^{2)}$ found a marked increase of blood flow in the posterior hypothalamic region during the arousal reaction, and also mentioned that the medial thalamic nuclei showed only slight and variable changes in blood flow. However, no comparison can be made between their results and ours, because we made no measurement on the hypothalamus, and they did not mention about the other thalamic nuclei.

A number of investigators ${ }^{4,8,10)}$ asserted that blood flow of individual parts of the brain is more likely to be regulated by the vasodilator effect of carbon dioxide than the neural vasomotor activity. On the other hand, it was shown by Ingvar ${ }^{4}$ that carbon dioxide content in the blood collected from the sagittal sinus increased on MRF stimulation. From these observations, it would be expected that the cerebral blood flow would increase on MRF stimulation. In reality, however, the experiments by Kanzow et al., Birzis and Tachibana and the present authors showed, contrary to the expectation, very low vascular responsiveness of the cortex. The discrepancy between the positive result by Ingvar and the negative ones by the other authors seems to be due to the difference of the methods used. If this is the case, further critical evaluation of these methods would be necessary. As has been mentioned above, the possibility of destroying the cortical regulatory mechanism and contamination with subcortical blood flow have been considered with the direct method employed by Ingvar. As to the indirect methods used by the other investigators, changes in the physical properties of the brain tissue other than blood flow may have some influence upon the measurement, and inasmuch as changes in the temperature and volume of the tissue are apt to occur more easily in the cortex than in the subcortical structure, these factors should be taken into consideration in evaluation of the indirect methods. For example, in the present method in which the cooling effect of the blood flow upon the electrical resistance of the thermistor is utilized, a flow increase as measured by this method would be cancelled out by a rise in temperature which might be caused by a flow increase when the temperature of the cortex is lower than that of the subcortex ${ }^{7}$. This factor is, however, almost negligible when the current heating of the thermistor is sufficiently strong. In our method the temperature of the thermistor was higher by about ten degrees than that of the brain tissue, and the cortex was well protected from cooling, so that the above factor cannot be responsible for the absence of the flow response in the cortex. The minor importance of this factor can be seen also in the fact that a remarkable flow response could be obtained by one and the same method from the 
cortex of an intact animal. It is conceivable that indirect methods such as ours can yield erroneous results when the sensing element happens to be displaced by some mechanical disturbance. For example, a false response imitating a flow increase would ensue when the sensing element is displaced from a heated locus into a less heated place. However, such a source of error would make a flow response appear more pronounced, but provide no explanation of the abovementioned absence of flow responses in the cortex.

We were interested in the dicrotic nature of the thalamic flow response and tried to analyze it under the assumption that the two components in the response were produced by different mechanisms. In order to see whether or not the recovery cycles of the two components were different from each other, two reticular stimuli were applied at different intervals, but the attempt was unsuccessful, because both components appeared to become smaller in a similar manner as the interval of stimuli was decreased. The duration of the second component was generally as long as one minute or so. Therefore it did not seem to have any relation to the spontaneous fluctuation which appeared sometimes with a so regular rhythm of about 8 cycles per minute. Thus, the mechanism and physiological significance of the flow pattern as revealed by our method had to be left to further studies.

From our experiments some comments will be made on the relationship between the pressor response and EEG arousal. Concerning the adrenalineinduced arousal, Baust et al. ${ }^{1}$ ) advanced a hypothesis that an increase in blood pressure activates pressure-sensitive neurons in the reticular system to cause EEG arousal. In the encéphale isolé preparation, which failed to show any pressor response, the EEG arousal could be induced by MRF stimulation as markedly as in the intact animal, and this fact suggests that at least in the case of the arousal induced by natural or electrical stimulus, the pressor-sensitive neurons within the brain stem have nothing to do with the arousal.

\section{Acknowledgement}

The authors wish to thank Dr. K. Motokawa for his invaluable discussion and suggestion throughout the course of the experiment and the proparation of the manuscript. Thanks are also due to Mr. M. Kurama for technical assistance.

\section{References}

1) Baust, W., Hiemczyk, H. \& Vieth, J., EEG clin. Neurophysiol., 1963, 15, 63.

2) Birzis, L. \& Tachibana, S., Exptl. Neurol., 1964, 9, 269.

3) Carlyle, A. \& Grayson, J., J. Physiol., 1956, 133, 10.

4) Ingvar, D. H., Reticular Formation of the Brain (H. Jasper ed.), Little, Brown and Co., Boston, 1958, p. 381.

5) Kanzow, E., Gregl, I. M., Held, U. P. \& Richtering, I., Pflüg. Arch. ges. Physiol., $1961,273,288$.

6) Kanzow, E. \& Krause, D., ibid., 1962, 274, 447. 
7) Ludwigs, N., ibid., 1954, 250, 35.

8) Schmidt, C. F., Amer. J. Physiol., 1935, 114, 447.

9) Suzuki, H. \& Tukahara, Y., Tohoku J. exp. Med., 1963, 81, 238.

10) Santha, K. \& Cipriani, A., Res. Publ. Ass. ment. Dis., 1938, 18, 346. 